\title{
DISTRIBUCIÓN DEL TEJÓN - MELES MELES (LINNEO, 1758) EN EL PARQUE NATURAL DE LA SIERRA DE MARIOLA (COMUNIDAD VALENCIANA)
}

\author{
Antonio Belda ${ }^{1}$ \\ Roque Belenguer ${ }^{2}$ \\ Benito Zaragozí \\ Vicente Ferri ${ }^{4}$ \\ ${ }^{1}$ Departamento de Ciencias de la Tierra y Medio Ambiente, ${ }^{2}$ Departamento de Ecología, \\ ${ }^{3}$ Instituto Interuniversitario de Geografía, ${ }^{4}$ Fundación C.V. Victoria Laporta Carbonell \\ antonio.belda@ua.es
}

\section{RESUMEN}

Este trabajo de investigación es pionero y original ya que nunca antes se ha realizado un estudio del tejón (Melesmeles) dentro del ámbito valenciano y más concretamente sobre la relación de su distribución con los factores geográficos que la condicionan en el Parque Natural Sierra de Mariola. Un mejor conocimiento de su ocupación en el espacio será de interés para la definición de medidas de gestión de fauna del Parque. En 2009, utilizando técnicas de fototrampeo se recopilaron 29.941 imágenes con algún contacto animal. De estas imágenes, el 0,16\% de las fotografías registradas son de tejón y se ha detectado su presencia en 6 de las 63 cuadrículas $\left(4 \mathrm{Km}^{2}\right)$ del Parque Natural Sierra de Mariola $(9,38 \%)$. Este estudio ha permitido integrar la información recopilada en campo con las bases de datos existentes para concluir que la situación del tejón en Sierra de Mariola no es preocupante.

Palabras clave: Distribución, Fototrampeo, Tejón, Paisaje, Parque Natural Sierra de Mariola.

\section{ABSTRACT}

The main objective of this research was to determine the distribution of the badger (Melesmeles) in the Sierra de Mariola Natural Park. A better knowledge of their distribution

Fecha de recepción: diciembre 2013.

Fecha de aceptación: noviembre 2014. 
will assist with the definition of management measures in the Natural Park. In 2009, using camera traps, 29,941 images were collected with any animal contact. From these images, the $0.16 \%$ of the photographs are registered with the badger and their presence was detected in 6 of the 63 grids $\left(4 \mathrm{Km}^{2}\right)$ of Sierra de Mariola (9,38\%). This study has allowed us to integrate the information collected in the field with existing data bases. We conclude that the situation of badger in Sierra de Mariola is not of concern.

Keywords: camera trap, distribution, tejón, landscape, Sierra de Mariola.

\section{INTRODUCCIÓN}

El tejón euroasiático (género Meles) es una especie carnívora territorial que habita nuestro planeta desde la antigüedad, estimándose por los palenteólogos, que ya tenía las características actuales hace 4 millones de años considerándose que su dentición ha sido lo que más ha evolucionado. El tejón tiene una amplia distribución (Figura 1) a lo largo de la región paleártica y comprende desde la Península Ibérica hasta Japón en sentido longitudinal, y desde Escandinavia y Siberia al Norte hasta Palestina, Irán, Sur de China y Tíbet en sentido latitudinal. Además, también está presente en las islas mediterráneas de Creta y Rodas, excepto en Cerdeña, Córcega y Sicilia, y en las islas de Reino Unido e Irlanda (Revilla y Palomares, 2002b; Del Cerro, 2011).

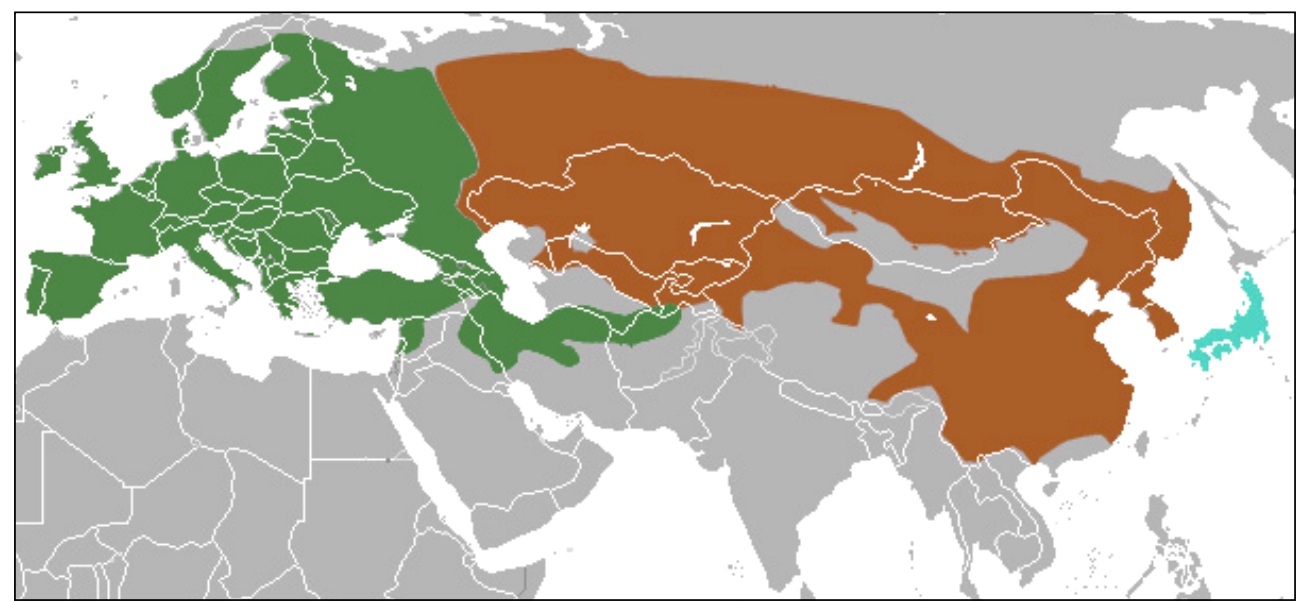

Verde $=$ Tejón Europeo (Melesmeles), Rojo= Tejón Asiático (Melesleucurus), Verde claro = Tejón Japonés (Melesanakuma) Fuente: Dominio Público. Cortesía de Paolo Conti (2007).

Desde el punto de vista biológico, el tejón en Europa ha sido ampliamente estudiado. Su hábitat comprende los bosques caducifolios, los pastos naturales, los humedales y zonas ribereñas, las formaciones de bosque con áreas de cultivo tradicional, zonas semi-desérticas e incluso zonas suburbanas (Revilla et al., 2000; Molina-Vacas, et al., 2009a). El área de 
campeo puede variar mucho entre individuos y en función de la productividad de los hábitats ocupados, siendo el tamaño medio de 525 hectáreas en el Suroeste de la Península Ibérica (Rodríguez, 1996). La presencia de tejón se ve favorecida por la cobertura arbórea y arbustiva en el fragmento, la existencia de corredores naturales y los cultivos herbáceos por debajo de un umbral del 50\% del área circundante (Palomo y Gisbert, 2002; Pereira, 2011).

En cuanto a la dieta,el tejón incluye una gran variedad de frutos (Pigozzi, 1991), conejos (Martín et al., 1995), pájaros (Hounsome y Delahay, 2005), insectos (Murdoch y Buyandelger, 2010) o gusanos de tierra (Kruuk y Parish, 1981). Por este motivo, algunos autores sugieren que su dieta es oportunista y se alimenta según los recursos (Neal y Cheeseman, 1996; Revilla y Palomares, 2002a), mientras que otros sugieren una dieta especializada (Kruuk y Parish, 1981). Además, en otros estudios se sugiere que la dieta puede estar correlacionada con la latitud: a mayor latitud, el porcentaje de proteínas en la dieta es mayor. También depende de la estacionalidad (Goszczyński et al., 2000; Hounsome y Delahay, 2005). Sin embargo, la variación latitudinal no es un buen predictor de la densidad de población, ya que las características y recursos de cada hábitat pueden afectar al tamaño poblacional (Del Cerro, 2011).

Esta especie se encuentra en regresión debido principalmente a la caza ilegal (Belda et al., 2008), a las perturbaciones antrópicas y a la destrucción del hábitat con la consiguiente reducción de sus biotipos (Belda et al., 2009; Lara-Romero et al., 2012).La especie de tejón M. meles (Europa y Sudoeste de Asia) se encuentra clasificada con la categoría Least Concern (LC, menor protección) de acuerdo con la Unión Internacional por la Conservación de la Naturaleza y de los Recursos Naturales (IUCN) desde 1996, debido a su tendencia poblacional estable. Además, dicha especie se incluye en el Apéndice III del Convenio de Berna desde 1979 (http://conventions.coe.int/Treaty/FR/Treaties/Html/104-3.htm). Por otro lado, a nivel nacional, el tejón euroasiático está protegido por los siguientes países España, Portugal, Italia, Bélgica, Países Bajos, Albania, Grecia, Estonia, Luxemburgo, Hungría, Reino Unido e Irlanda (Griffiths y Thomas 1997).

El uso de modelos de distribución y abundancia se está mostrando eficaz en el diseño de planes de conservación de fauna (Ferrier, 2002). Estos modelos permiten avanzar en la comprensión de los patrones geográficos de biodiversidad (Graham et al., 2006).

En 2009, se creó un grupo de investigación que, realiza estudios de seguimiento de mamíferos en el Parque Natural de la Sierra de Mariola. La serie de trabajos desarrollados en el seno de este grupo reflejan la importancia de la conservación de los espacios agroforestales tradicionales y las poblaciones de vertebrados con elevado interés ecológico y cinegético (López et al., 1991; Belenguer et al., 2011). Además de la acción del hombre, la abundancia de predadores en un espacio territorial cada vez más restringido y transformado puede propiciar una importante reducción de la disponibilidad de hábitat para esta especie. Los resultados de estudios como el presente pueden servir para mejorar los planes de gestión del parque, ayudando a incrementar la calidad del hábitat para este mustélido (Ballesteros, 1998; Chase, 2000, Angulo, 2003; Lombardi, 2003), y permitiendo a los gestores de los cotos de caza una toma de decisiones rápida y efectiva (Mathevet \& Tamisier, 2002; Stoate et al., 2004).

El objetivo principal de este trabajo es realizar una estimación de la abundancia y distribución del tejón en el Parque Natural de la Sierra de Mariola. Ésta es una contribución 
novedosa ya que hasta este momento no existe ninguna publicación sobre esta especie en la zona de estudio. Finalmente, se plantea una primera explicación de los factores geográficos locales que inciden en la distribución de esta especie.

\section{II. ÁREA DE ESTUDIO}

La Sierra de Mariola es una formación montañosa situada al sureste de la Península Ibérica (Figura 2), concretamente en la Comunidad Valenciana. Este relieve es a la vez frontera y nexo de las comarcas de l'Alcoià, la Valld'Albaida y el Comtat, abarcando una superficie total superior a 17.500 hectáreas, lo que lo convierte en uno de los parques naturales más extensos de la Comunidad Valenciana. Con un clima típicamente mediterráneo, con temperaturas suaves, lluvias concentradas en primavera y otoño y un destacado periodo seco en verano, las temperaturas medias anuales oscilan entre los 13 y $16^{\circ} \mathrm{C}$. La vegetación climácica es latípica del Termótipo Mesomediterráneo y Ombrotipo Subhúmedo, dando lugar a carrascales (asociación Hederohelicis-Quercetumrotundifoliae subas. ulicerosumparviflorae) (Belda et al., 2009).

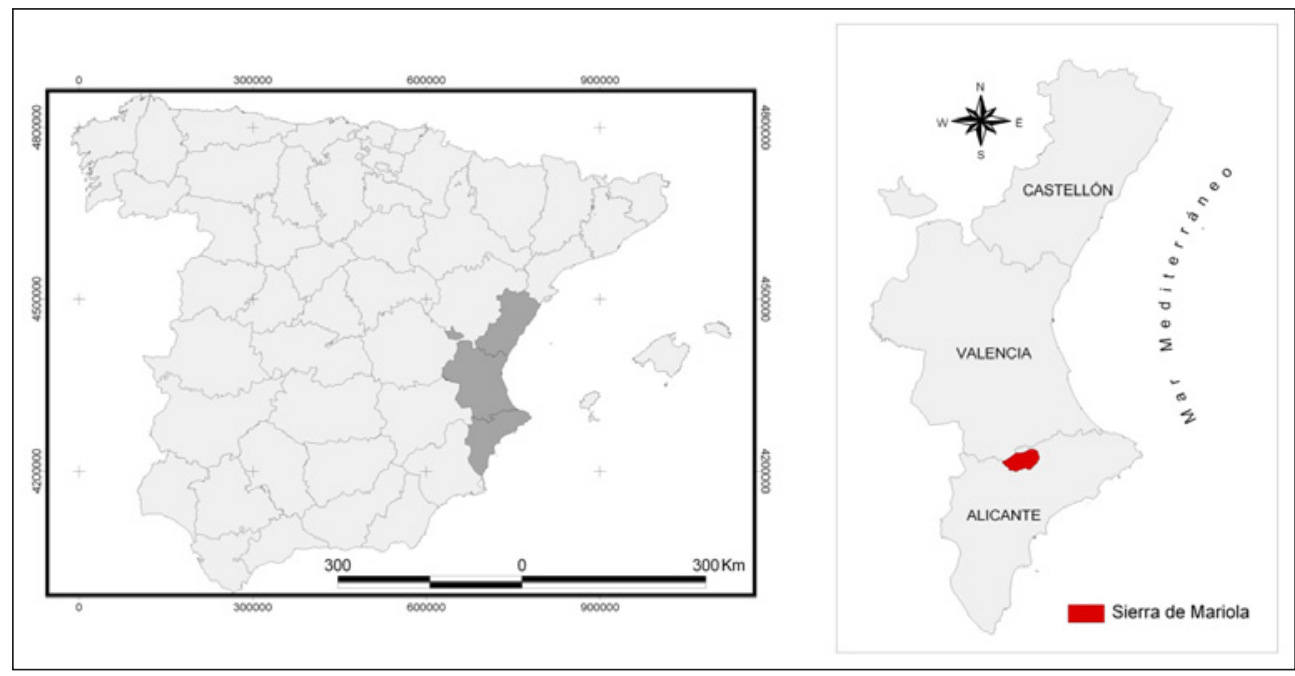

\section{MATERIAL Y MÉTODOS}

La metodología empleada para determinar la presencia/ausencia y la abundancia relativa del tejón se ha basado en la técnica conocida como fototrampeo. El trampeo fotográfico es una práctica no invasiva utilizada en diversas áreas de conocimiento, como la investigación de la fauna silvestre, la gestión de especies de caza, el control de especies o la educación ambiental. Esta es una actividad en auge debido a la reciente incorporación y abaratamiento de diversas tecnologías aplicadas a equipos fotográficos automatizados, 
como los sensores de movimiento, las cámaras digitales, las tarjetas de memoria compacta, los flash de infrarrojos, las baterías de larga duración, etc. La fotografía realizada con estos equipos no pretende tener un encuadre o una calidad técnica perfecta, sino que proporciona la frescura de saber que ese instante pertenece a un ejemplar salvaje y único sin las perturbaciones de la presencia humana. Estos equipos autónomos pueden ser colocados en lugares remotos durante varias semanas, incluso meses, sin tener que realizar mantenimiento alguno, por lo que se configuran como un recurso para la investigación de incomparable utilidad. Las imágenes que están proporcionando estos equipos permiten conocer no solo la presencia de algunas especies, sino obtener estimas de su frecuencia y densidad, así como la identificación de individuos a través del diseño del pelaje, las manchas de identificación, etc., lo que proporciona a los investigadores una información muy valiosa (Belda et al., 2009). En la Figura 3 se puede ver un ejemplo de imagen tomada con cámaras de fototrampeo.

Figura 3

DETALLE DEL TEJÓN (MELES MELES) MEDIANTE TRAMPEO FOTOGRÁFICO

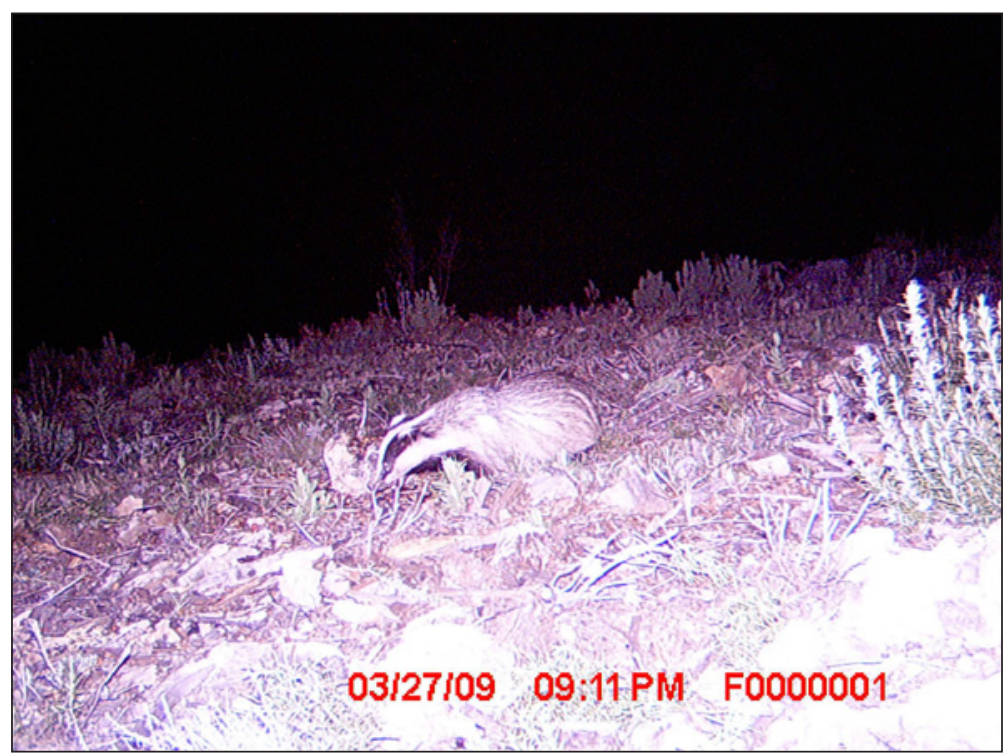

La zona de estudio se dividió en 63 cuadrículas con una extensión de $4 \mathrm{~km}^{2}$ cada una. Se colocaron dos cámaras de fototrampeo del modelo StealthCam-IR® en cada una de las celdas durante un período de 2 semanas. Los dispositivos se programaron para realizar 3 fotografías consecutivas con un período de reposo de 5 minutos entre cada ráfaga. La información se ha almacenado en una tarjeta de memoria SD de 2 GB. Las unidades han sido equipadas con un sistema de alimentación externo, constituido por una batería de $12 \mathrm{~V}$ y los cables de alimentación. La distancia entre los equipos de fototrampeo ha sido al menos de 200 metros. El periodo de muestreo se prolongó desde agosto de 2008 hasta septiembre de 2009. Las cámaras fueron instaladas en los lugares más propicios para interceptar el paso 
del animal. Se empleó como cebo una mezcla compuesta por $1 / 2 \mathrm{~kg}$ de maíz, $1 / 2 \mathrm{~kg}$ de trigo, $1 / 2 \mathrm{~kg}$ de pienso de perro, $1 / 2 \mathrm{~kg}$ de almendras y una lata de sardinas con aceite de oliva. Las cámaras registraron información relacionada con la presión atmosférica, temperatura, fase lunar, fecha y hora.

Todas las fotografías capturadas fueron almacenadas en un mismo ordenador portátil y analizadas con un software propio para la gestión de imágenes de fototrampeo. Este software permite crear bases de datos geográficas a partir de las propias imágenes, para posteriormente explorar esta información en un SIG, crear informes y cartografía (Martínez et al., 2011).

\section{RESULTADOS Y DISCUSIÓN}

Existen diferentes métodos para monitorear la biodiversidad los cuales se seleccionan de acuerdo a diferentes criterios. Dos de los métodos más utilizados para el monitoreo de mamíferos medianos y grandes son las trampas de huella y las cámaras de fototrampeo, ambos son métodos no invasivos que permiten identificar qué especies se encuentran en un área determinada, monitorear abundancia relativa y absoluta de especies, además de esto, el fototrampeo se ha utilizado para estudiar patrones de actividad (Lyra-Jorge et al., 2008). Así mismo, la metodología presenta varias ventajas, entre las cuales se destacan, que una vez fotografiado el individuo este puede ser identificado y que el equipo no tiene que ser monitoreado constantemente (McCullough et al., 2002). Así, esta metodología ha permitido integrar en una base de datos geográfica la información recopilada en campo sobre la fauna silvestre. A partir de dicha información, se ha realizado una estimación de la biodiversidad animal dentro del Parque Natural de la Sierra de Mariola. En total se han recopilado 72.347 imágenes (más de $93 \mathrm{~Gb}$ ) de las cuales 29.941 muestran algún contacto animal dentro de las cuadrículas definidas. Entre estas fotografías se han registrado 48 en las cuales está presente el tejón. Esto supone el 0,16\% del número de total de fotografías válidas. Por otro lado, la garduña ha sido detectada en 6 cuadrículas y supone un 9,38\% respecto del total de cuadrículas $(n=63)$. Cabe mencionar que se comprobó el comportamiento nocturno de esta especie, puesto que todas las fotografías capturadas se tomaron de noche. Estas estadísticas y cartografía (ver Figura 4) se generaron de manera semiautomática mediante el software descrito en el apartado anterior. No obstante, los resultados obtenidos podrían completarse con otros métodos si se desee obtener valores cuantitativos de la abundancia de la especie en el medio (Del Cerro, 2011).

La distribución del tejón según los usos del suelo muestra que las mayores abundancias se dan en la unidad de cultivos de regadío seguida de las zonas con vegetación riparia. El regadío solamente ocupa un $1 \%$ de la superficie del parque y se caracteriza por la tecnificación del regadío en frutales, olivos, viñas y huertos, situados en zonas llanas. Por otro lado, también aparece en algunos puntos de los usos de secano (24\%), que está formada por pequeñas parcelas de olivos y almendros en las zonas más elevadas, mientras que en los valles predominan el girasol y el trigo. Las cuadrículas con vegetación natural y cultivos abandonados son las que acogen un número menor de presencias. Así, los usos naturales ocupan la mayor superficie del parque $(67 \%)$ y se caracteriza por la presencia de pinares maduros, encinares y coscojares con abundante matorral. En cambio, la unidad de cultivos 
abandonados, solamente cuenta con una superficie del 3\% del parque (Figura 4). Se trata de parcelas de almendros y olivos con abundante matorral que hace años se abandonaron debido a su baja rentabilidad. En la Figura 4 se pueden apreciar, a grandes rasgos, tres áreas con mayor presencia de esta especie, una en su sector suroeste que coincide con el curso del rio Vinalopó y su vegetación de ribera asociada, junto con los cultivos de secano y regadío adyacentes. Por otra parte, hay otras dos zonas,en el sector norte-noreste, del parque con presencia de tejón que se caracterizan por pequeños barrancos insertados dentro de zonas con superficies forestales maduras que contienen pequeñas explotaciones agrarias (masías), en las cuales los tejones encuentran fácilmente refugio y alimento.

En otros estudios similares en ambientes mediterráneos, el tejón también aparece en baja medida, con 4 fotos y presencia en un 1,3\% de las unidades de muestreo. Así, el tejón es una especie que está en baja frecuencia o incluso no se detecta en estudios que utilizan el trampeo fotográfico. Es asumible que las limitaciones del fototrampeo se vean impuestas por el atrayente utilizado, pues es difícil conseguir uno que atraiga a todas las especies de carnívoros. Es por ello que puede ser apropiado combinar diferentes sustancias para conseguir fotografiar a un mayor número de especies de carnívoros, como por ejemplo, compuestos sintéticos combinados con cebos comerciales (Torre et al., 2009).

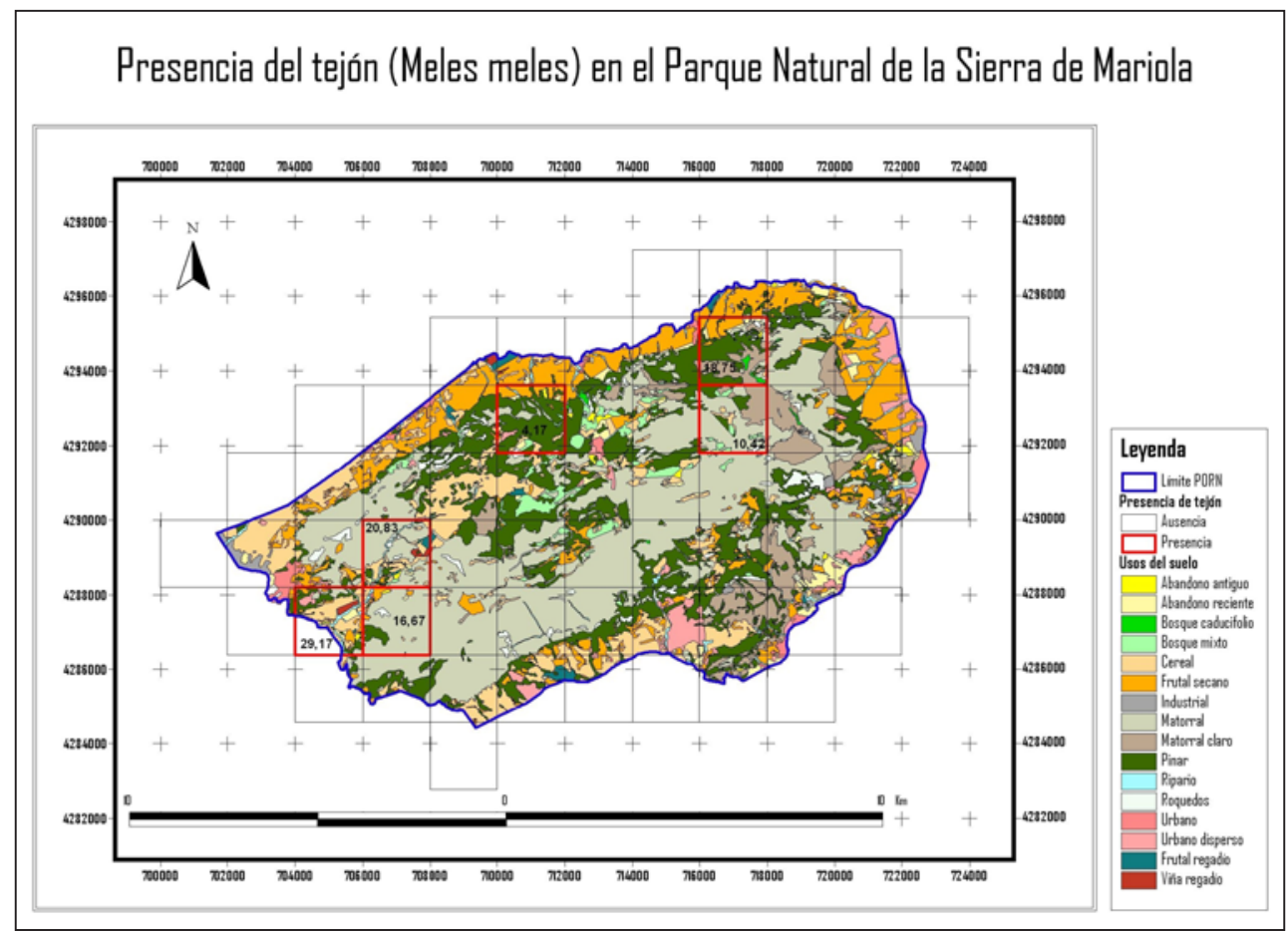


En Europa, se han encontrado bajas densidades de población en el área mediterránea (0.2-0.7ind. $/ \mathrm{km}^{2}$, sudoeste de España, Revilla y Palomares 1999) y en Centro de Europa (0.2 ind.$/ \mathrm{km}^{2}$, Polonia, Kowalczyket al., 2003) mientras que se describen muy altas densidades en las islas Británicas (2-38 ind./km², Johnson et al., 2002). Así, los tejones que habitan zonas densas forman grupos de muchos individuos que comparten una tejonera principal, ocupan un territorio muy reducido $\left(0.14 \mathrm{~km}^{2}\right.$, Cheeseman et al., 1981) y existe una jerarquía en las hembras reproductoras que lo constituyen (Woodroffe et al., 1995). En cambio, los tejones que conviven en zonas de baja densidad o densidad medias (Europa continental) forman grupos con una sola pareja reproductora y ocupan territorios amplios $\left(13 \mathrm{~km}^{2}\right.$, Kowalczyk et al., 2006). Por tanto, la organización social del tejón es más organizada y con menos flujo de migrantes en zonas de alta densidad que en zonas de baja densidad, ya que la finalidad de la migración de un tejón macho es encontrar recursos y/o aparearse con hembras receptivas no emparentadas con él. Se puede citar que un tejón en el Reino Unido se desplaza a una velocidad media de $1 \mathrm{~km} /$ día mientras que un tejón en Polonia es capaz de recorrer una media de $7 \mathrm{~km} /$ día (Woodroffe et al., 1995; Kowalczyk et al., 2006).

En algunos sectores de la Sierra de Mariola se han producido algunos episodios de cambios de uso del suelo considerables, especialmente por la transformación de uso natural en suelo urbano o incluso debido a los recurrentes incendios (Figura 4). Una primera interpretación de los datos obtenidos en este estudio muestra que los usos del suelo pueden estar muy relacionados con la abundancia relativa del tejón. Así, los cultivos que se encuentran intercalados en la unidad natural deben ser tenidos en cuenta en los planes de gestión que pretendan la conservación del tejón. La información proporcionada en esta prospección puede servir a los gestores del parque para realizar futuras actuaciones de ordenación y de mejora del hábitat.

Los estudios centrados en la conservación del tejón euroasiático en las zonas mediterráneas son escasos en la Península Ibérica (Molina-Vacas, et al., 2009b). Aun así, se ha detectado diferencias genéticas y de densidad poblacional entre la población de tejón y que ha sufrido el impacto de las infraestructuras viarias durante las últimas décadas (MolinaVacas et al. 2009a). Otras de las amenazas ha sido desarrollo de la agricultura y ganadería intensiva, durante un periodo que comprendió desde 1960 hasta 1980, reduciendo las zonas forestales en las áreas de pasto y los cultivos tradicionales en toda Europa, desde el Norte de Europa (Lankester et al., 1991) hasta el área mediterránea (Virgós et al., 2005). Solapándose con este periodo, también se produjo un incremento de infraestructuras viarias (carreteras y vías ferroviarias). La unión de estos dos fenómenos produce lo que se conoce como la fragmentación del hábitat, que es capaz de generar un posterior declive en el tamaño poblacional del tejón (Clarke et al., 1998). Por otro lado, el zorro, gato montés y gineta depredan sobre los ejemplares jóvenes, al igual que las grandes rapaces nocturnas y diurnas. La finalidad de los estudios del tejón euroasiático dentro del campo de la conservación es prevenir posibles declives en sus poblaciones causados a lo largo del tiempo o recientemente, mediante el control de la evolución del tamaño efectivo de sus especies y el análisis de la diversidad genética de sus poblaciones (Guisan et al., 2006; Lawler et al., 2006; Del Cerro, 2011).

Este trabajo es pionero puesto que proporciona y analiza datos hasta ahora no abordados en este territorio. De este modo, la información recopilada en campo junto con las bases de datos existentes permite concluir que la situación del tejón en Sierra de Mariola no 
es preocupante. Así, las imágenes capturadas mediante fototrampeo, junto con el software propuesto, permitirán en un corto periodo de tiempo que podamos aportar nuevos datos y explicaciones sobre las poblaciones de mamíferos que habitan la Sierra de Mariola. Además, la habilitación de pasos específicos para la fauna en las infraestructuras viales y el control del furtivismo son dos objetivos a alcanzar para controlar las altas tasas de mortalidad de tejones y favorecer el buen estado de conservación de sus poblaciones. Por otro lado, la continuación de este estudio pasa por analizar el resto de mamíferos que habitan el parque, comprobar su situación actual y la interacción entre distintas especies.

\section{AGRADECIMIENTOS}

Este proyecto ha sido financiado en parte por las ayudas de investigación del Instituto Alicantino de Cultura Juan Gil-Albert. Los autores también quieren agradecer la colaboración prestada por la Dirección y personal del Parque Natural de la Sierra de Mariola. Además de la información proporcionada por los gestores cinegéticos y la ayuda de la Fundación C.V. Victoria Laporta.

\section{BIBLIOGRAFÍA}

ANGULO, E. and VILLAFUERTE, R. (2003): «Modelling hunting strategies for the conservation of wild rabbit populations». Biological Conservation, 115: 291-301.

BALLESTEROS, F. (1998): Las especies de caza. Biología, ecología y conservación. Estudio y Gestión del Medio, Colección técnica, Oviedo. 316 pp.

BELDA, A.; ARQUES, J.; MARTÍNEZ, J.E.; PEIRÓ, V. and SEVA, E. (2009): «Análisis de la biodiversidad de fauna vertebrada en el Parque Natural de la Sierra de Mariola mediante fototrampeo». Mediterranea, 20: 9-32.

BELDA, A.; MARTÍNEZ, J.E.; PEIRÓ, V.; ARQUES, J.; SEVA, E. and JIMÉNEZ, D. (2008): «Métodos de caza tradicionales empleados en el Carrascal de la Font Roja». Mediterranea, 19: 9-34.

BELENGUER, R.; BELDA, A.; ARQUES, J.; MARTÍNEZ-PÉREZ, J.E. and ZARAGOZÍ, B. (2011): «Influencia de la estructura del paisaje en la comunidad de mamíferos del Parc Natural de la Serra de Mariola (Comunidad Valenciana). Una aproximación mediante fototrampeo». En X Congreso SECEM, 16. Málaga: Universidad de Málaga.

CHASE, L.C.; SCHUSLER, T.M. and DECKER, D.J. (2000): «Innovations in stakeholder management: what's the next step?» Wildlife Society Bulletin, 28: 208-217.

CHEESEMAN, C.L.; JONES, G.W. and GALLAGHER, J. (1981): «The population structure, density and prevalance of tuberculosis (Mycobacterium bovis) in badgers (MelesMeles) from four areas in south-west England». Journal of Applied Ecology. 18, 795-804.

CLARKE, G.P.; WHITE, P.C.L. and HARRIS, S. (1998): «Effects of roads on badger MelesMeles populations in south-west England». Biological Conservation, 86, 117-124.

DEL CERRO, I. (2011): Filogeografía, clarificación taxonómica y estructura poblacional del tejón Eurasiático (Melesspp.). Tesis doctoral, Universidad Autónoma de Barcelona. $287 \mathrm{pp}$. 
FERRIER, S. (2002): «Mapping spatial pattern in biodiversity for regional conservation planning: where to from here?» Systematic Biology, 51: 331-363.

GOSZCZYŃSKI, J.; JĘDRZEJEWSKA, G. and JĘDRZEJEWSKI, W. (2000): «Diet composition of badgers (MelesMeles) in a pristine forest and rural habitats of Poland compared to other European populations». Journal of Zoology, 250, 495-505.

GRAHAM, C.H.; MORITZ, C. and WILLIAMS, S.E. (2006): «Habitat history improves prediction of biodiversity in a rainforest fauna». Proceedings of the Natural Academy of Science of USA, 103: 632-636.

GRIFFITHS, H.I. and THOMAS, D.H. (1997): «The Conservation and Management of the European Badger (Melesmeles).» Nature and Environment, No. 90. Council of Europe, Strasbourg, France.

GUISAN, A.; BROENNIMANN, O.; ENGLER, R.; VUST, M.; YOCCOZ, N.G.; LEHMANN, A. and ZIMMERMANN, N.E. (2006): «Using niche-based models to improve the sampling of rare species». Conservation Biology, 20(2): 501-511.

HOUNSOME, T. and DELAHAY, R. (2005): «Birds in the diet of the Eurasian badger Melesmeles: a review and meta-analysis». Mammal Review, 35, 199-209.

JOHNSON, D.D.P.; JETZ, W. and MACDONALD, D.W. (2002): «Environmental correlates of badger social spacing across Europe». Journal of Biogeography, 29, 411-425.

KOWALCZYK, R.; ZALEWSKI, A. and BOGUMIŁA, J. (2006): «Daily movement and territory use by badgers Melesmelesin Białowieża Primeval Forest, Poland». Wildlife Biology 12, 385-391.

KOWALCZYK, R.; ZALEWSKI, A. and JEDDRZEJEWSKA, B. (2003): «Spatial organization and demography of badgers MelesMelesin Białowieża Forest (Poland) and the influence of earthworms on badger densities in Europe». Canadian Journal of Zoology, $81,74-87$.

KRUUK, H. and PARISH, T. (1981): «Feeding specialization of the European badger Melesmeles in Scotland». Journal of Animal Ecology, 50, 773-788.

LANKESTER, K.; VAN APELDOORN, R. and MEELIS, E. (1991): «Management perspectives for populations of the Eurasian badger (Melesmeles) in a fragmented landscape». Journal of Applied Ecology, 28, 561-573.

LAWLER, J.J.; WHITE, D.; NEILSON, R.P. and BLAUSTEIN, A.R. (2006). «Predicting climate-induced range shifts: model differences and model reliability». Global Change Biology, 12: 1568-1584.

LOMBARDI, L.; FERNÁNDEZ, N.; MORENO, S. and VILLAFUERTE, R. (2003): «Habitat-related differences in rabbit (Oryctolaguscuniculus) abundance, distribution and activity». Journal of Mammalogy, 84: 26-36.

LÓPEZ, G.; RICO, L. and MARTÍN, C. (1991): Cuadernos de la naturaleza: elsvertebrats terrestres de la comarca d'Alacant. Caixad'Estalvis Provincial d'Alacant. 179 pp.

LYRA-JORGE, M.C.; CIOCHETI, G.; PIVELLO, V.R. and MEIRELLES, S.T. (2008): «Comparing methods for sampling large- and medium-sized mammals: Camera traps and track plots». European Journal of Wildlife Research, 54: 739-744.

MARTÍN, R.; RODRÍGUEZ,A. and DELIBES, M. (1995): «Local feeding specialization by badgers (Melesmeles) in a Mediterranean environment». Oecologia, 101, 45-50. 
MARTÍNEZ, J.E.; ZARAGOZÍ, B.M.; BELDA, A.; NAVARRO, J.T. and PEIRÓ, V. (2011): Creación de un software para el almacenamiento automático y gestión de imágenes obtenidas por fototrampeo. En X Congreso SECEM, Málaga, Universidad de Málaga.

MATHEVET, R. and TAMISIER, A. (2002): «Creation of a nature reserve, its effects on hunting management and waterfowl distribution in the Camargue (southern France)». Biodiversity and Conservation, 11: 509-519.

MCCULLOUGH, D.R.; PEI, K.C. and WANG, Y. (2002): «Home range, activity patterns, and habitat relations of Reeeves' muntjacs in Taiwan». En: YASUDA, M. and KAWAKAMI, K. «New method of monitoring remote wildlife via the internet». Ecological Research, 17:119-124».

MOLINA-VACAS, G.; BONET-ARBOLÍ, V. and RAFART-PLAZA, E. (2009a): «Spatial ecology of European badgers (Melesmeles) in Mediterranean habitats of the North-Eastern Iberian peninsula I: home range, spatial distribution and social organization». Vie et milieu - life and environment, 59, 227-236.

MOLINA-VACAS, G.; BONET-ARBOLÍ, V. and RAFART-PLAZA, E. (2009b): «Spatial ecology of European badgers (Melesmeles) in Mediterranean habitats of the North-Eastern Iberian peninsula II: Habitat selection. Vie et milieu - life and environment, 59, 237246.

MURDOCH, J.D. and BUYANDELGER, S. (2010): «An account of badger diet in an arid steppe region of Mongolia». Journal of Arid Environments 74, 1348-1350.

NEAL, E.G. and CHEESEMAN, C. (1996): Badgers. T. \& A. D. Poyser, London.

PALOMO, L. J. and GISBERT, J. (2002): Atlas de los mamíferos terrestres de España. Dirección General de Conservación de la Naturaleza- SECEM-SECEMU, Madrid, 564 pp.

PEREIRA, M.A. (2011): Mamíferos carnívoros en un paisaje agrícola: distribución, selección de hábitat y patrones de movimiento. Tesis doctoral, Universidad de Granada. 158 pp.

PIGOZZI, G. (1991): «Thediet of the Europeanbadger in a Mediterraneancoastalarea». Acta Theriologica, 36, 293-306.

REVILLA, E. and PALOMARES, F. (1999): «Changes in the behaviour of a male Eurasian badger: evidence in favour of the anti-kleptogamy hypothesis?». ActaTheriologica, 44, 471-476.

REVILLA, E. and PALOMARES, F. (2002a): «Does local feeding specialization exist in Eurasian badgers?». Canadian Journal of Zoology, 80, 83-93.

REVILLA, E. and PALOMARES, F. (2002b): «Spatial organization, group living and ecological correlates in low-density populations of Eurasian badgers, Melesmeles». Journal of Animal Ecology, 71, 497-512.

REVILLA, E.; PALOMARES, F. and DELIBES, M. (2000): «Defining key hábitats for low density populations of Eurasian badgers in Mediterranean environments». Biological Conservation, 95, 269-277.

RODRÍGUEZ, A. (1996): «Space use and activity in a mediterranean population of badgers Melesmeles». Acta Theriologica, 41, 59-72.

STOATE, C.; HENDERSON, I.G. and PARISH, D.M. (2004): «Development of an agrinvironment scheme option: seed-bearing crops for farmland birds». Ibis, 146: 203-209. 
TORRE, I.; RIBAS, A. and ARRIZABALAGA, A. (2009): «Estudio de la comunidad de carnívoros del P. N. Del Montseny (Catalunya) mediante trampeo fotográfico». Galemys 21 ( $\mathrm{n}^{\mathrm{o}}$ especial): 165-180.

VIRGÓS, E.; REVILLA, E. and MANGAS, J.G. (2005): Factores que determinan la distribución y abundancia del tejón (Melesmeles) a escala regional. En: Ecología y conservación del tejón en ecosistemas mediterráneos. Eds: Virgós, E.; Revilla, E.; Mangas, J.G. and Domingo-Roura, X. págs. 149-172. Sociedad Española para la Conservación y Estudio de los Mamíferos, Màlaga.

WOODROFFE, R.; MACDONALD, D.W. and DA SILVA, J. (1995): «Dispersal and philopatry in the European badger (Melesmeles)». Journal of Zoology, 237, 227-239. 